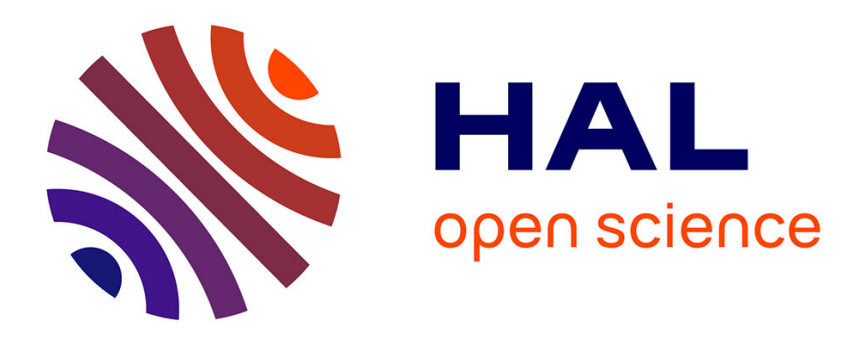

\title{
Mathematical modeling of piezomagnetoelectric thin plates
}

\author{
Thibaut Weller, Christian Licht
}

\section{To cite this version:}

Thibaut Weller, Christian Licht. Mathematical modeling of piezomagnetoelectric thin plates. European Journal of Mechanics - A/Solids, 2010, 29, pp.928-937. 10.1016/j.euromechsol.2010.06.002 . hal-00682985

\section{HAL Id: hal-00682985 \\ https://hal.science/hal-00682985}

Submitted on 27 Mar 2012

HAL is a multi-disciplinary open access archive for the deposit and dissemination of scientific research documents, whether they are published or not. The documents may come from teaching and research institutions in France or abroad, or from public or private research centers.
L'archive ouverte pluridisciplinaire HAL, est destinée au dépôt et à la diffusion de documents scientifiques de niveau recherche, publiés ou non, émanant des établissements d'enseignement et de recherche français ou étrangers, des laboratoires publics ou privés. 


\title{
Mathematical modeling of piezomagnetoelectric thin plates
}

\author{
Thibaut Weller, Christian Licht \\ Laboratoire de Mécanique et Génie Civil, \\ cc 048, Université Montpellier II, \\ 34095 Montpellier Cedex 5, France
}

\begin{abstract}
We present the outline of the rigorous mathematical derivation of linearized piezomagnetoelectric thin plate models and focus on the results it leads to. It is in particular shown how four different models emerge from theoretical tools. New mixed 'senso-actuator' and 'actuato-sensor' behaviors appear. Moreover, the influence of all the 45 crystal symmetry classes on our models is described. We point out the extremely important structural switch-off phenomenon for which the electromagnetoelastic coupling disappear for some specific crystal classes.
\end{abstract}

\section{Introduction}

The wide range of applicability of multiphysical materials has generated a vast field of researches aiming at a better understanding of smart devices (see [4], [7] and [17] for example). However, even if different modelings have already been presented in the literature (we refer the reader to [1], [6], [18] for example), a theoretical understanding of the difference between sensing and actuation is still to improve. Moreover the role that plays the crystal class of the genuine material constituting the device on its behavior has never been achieved, at the authors knowledge. Indeed, a rigorous theoretical investigation of such structures is quite recent (see [10], [11], [12], [15] and references quoted therein). Here, taking advantage of the multiscaled shape of MEMS plates of electromagneticoelastic nature, we present the results of their rigorous mathematical derivation that has been carried out in [13]. The models emerge through the study of the asymptotic behavior of a three dimensional body when its thickness is considered as a parameter whose aim is to tend to zero. Considering piezoelectric plates (see [10], [11], [12] and [15]), we have sharply shown how the electric boundary conditions are connected to two different models linked to sensing or actuation. Moreover, when looking at the influence of the crystalline symmetries of the material, it is possible to show that piezoelectric coupling may vanish at the limit leading to the striking result that a piezoelectric material can be no more piezoelectric when used as a plate-like body (see [16]). We have then enlightened what can be called a 'structural switch off'. Smart rods modeling has also been undertaken in [14] in which this structural switch off also appears. In the piezomagnetoelectric plates situation which is carried out here, the same 
asymptotic method leads to four different models in which sensing and actuation may be mixed. This makes possible the modeling of electrically commanded magnetic devices and of magnetically commanded electric ones, which is of considerable interest in the development of non-volatile magnetic random access memories.

Here, we shortly present our models in order to share results whose interest is from our point of view outside the sole Applied Mathematics field. Starting from a general three-dimensional piezomagnetoelectric problem denoted by $\mathcal{P}_{3 D}$, we outline the method that leads to simplified but accurate plate models denoted by $\mathcal{P}_{2 D}$ because they are two-dimensional in essence. The ground of the method is to view the thickness of the plate as a small normalizing parameter which tends to zero. In particular, it can be shown that the four limit generalized kinematics do not have the same number of variables. Moreover, we investigate the influence of crystalline symmetries on the properties of our models and show that some crystal classes lead to the striking structural switch off evoked earlier: even if the material is piezomagnetoelectric, it is not anymore the case for the thin plate. More precisely, this switch off does not depends only on the crystal class of the piezomagnetoelectric material that constitutes the plate but also on the electric boundary conditions, i.e. crystal symmetries do not have the same influence on sensors, actuators and mixed senso-actuators or actuato-sensors.

Finally, we give an example of our results in the case of a 222 symmetry class material.

\section{Theoretical considerations}

Let us first recall the basic equations governing the behavior of piezomagnetoelectric continua. It is the starting point of problems that can either be of mathematical or numerical nature.

\subsection{Basic piezomagnetoelectric equations}

Latin lower indexes run from 1 to 3 and the lower index ',$i{ }^{\prime}$ stands for the derivation with respect to the $i^{t h}$ coordinate. Moreover, the convention of summation over repeated indexes is understood.

The equilibrium of a piezomagnetoelectric body whose reference configuration is a $3 D$ domain $\Omega$ with boundary $S$ leads to:

$$
\begin{aligned}
\sigma_{i j, j}+f_{i} & =0, \quad D_{i, i}=0, \quad B_{i, i}=0 \\
\operatorname{rot} H & =j, \quad \operatorname{rot} E=0 .
\end{aligned}
$$

If the current density $j$ vanishes in $\Omega$ then the magnetic field $H$ derives from a magnetic scalar potential $\phi$, as the electric field $E$ which derives from an electric scalar potential $\varphi$. In the equations above, $f_{i}$ are the mechanical body force components, while $\sigma_{i j}, D_{i}$ and $B_{i}$ respectively stand for the Cauchy stress tensor, the electric and the magnetic inductions components. These latter components are related to those of small strain tensor $e_{i j}(u)=\frac{1}{2}\left(u_{i, j}+u_{j, i}\right)$ (u denotes the displacement vector field), of electric and magnetic vector fields $E_{i}=-\varphi_{, i}$ and $H_{i}=-\phi_{, i}$ by the constitutive equations (see [13] for example): 


$$
\begin{aligned}
\sigma_{i j} & =\mathrm{a}_{i j k h} e_{k h}(u)-\mathrm{b}_{k i j} \varphi_{k}-\mathrm{c}_{k i j} \phi_{k} \\
D_{i} & =\mathrm{b}_{i j k} e_{j k}(u)+\mathrm{d}_{i j} \varphi_{j}+\mathrm{e}_{i j} \phi_{j}, \\
B_{i} & =\mathrm{c}_{i j k} e_{j k}(u)+\mathrm{e}_{j i} \varphi_{j}+\mathrm{f}_{i j} \phi_{j},
\end{aligned}
$$

In this expression, $\mathrm{a}_{i j k h}, \mathrm{~b}_{i j k}, \mathrm{c}_{i j k}, \mathrm{~d}_{i j}, \mathrm{e}_{i j}$ and $\mathrm{f}_{i j}$ denote the elastic, piezoelectric, piezomagnetic, dielectric, electromagnetic coupling and magnetic permeability material constants, respectively.

Remark 1. It is possible to define an operator $M: \mathbb{R}^{12} \longrightarrow \mathbb{R}^{12}$ governing the constitutive law (2) by ${ }^{1}$

$$
\left(\begin{array}{l}
\sigma \\
D \\
B
\end{array}\right)=M\left(\begin{array}{c}
e(u) \\
\nabla \varphi \\
\nabla \phi
\end{array}\right)
$$

The triplet $\left(\begin{array}{c}\sigma \\ D \\ B\end{array}\right)$ is called the generalized stress, while $\left(\begin{array}{c}e(u) \\ \nabla \varphi \\ \nabla \phi\end{array}\right)$ is the generalized strain.

Most of the time, the mapping (2) is seen in a $12 \times 12$ matrix-form representation. In this direction, we introduce

$$
M=\left(\begin{array}{ccc}
a_{I J} & -b_{K l} & -c_{K l} \\
b_{l K} & d_{m n} & e_{m n} \\
c_{l K} & e_{n m} & f_{m n}
\end{array}\right)
$$

with $d_{m n}=\mathrm{d}_{m n}, e_{m n}=\mathrm{e}_{m n}, f_{m n}=\mathrm{f}_{m n}$ and where indexes $l, m$ and $n$ take their values in $\{1,2,3\}$ while $I, J$ and $K$ satisfy the Voigt contraction convention, taking their values in $\{1,2,3,4,5,6\}$. We recall that the Voigt contraction convention is a mapping which associates to a couple of indexes $(i, j)$ a sole index $I$ such that

\begin{tabular}{|c|c|c|c|c|c|c|}
\hline$(i, j)$ & $(1,1)$ & $(2,2)$ & $(3,3)$ & $(2,3)$ & $(3,1)$ & $(1,2)$ \\
\hline$I$ & 1 & 2 & 3 & 4 & 5 & 6 \\
\hline
\end{tabular}

Thus, the elastic tensor a can be seen as a $6 \times 6$ real matrix which is written in another font by $a$. In the same way, the piezoelectric and the piezomagnetic tensors $\mathrm{b}$ and $\mathrm{c}$ take the form of $6 \times 3$ real matrices respectively denoted by $b$ and $c$. However, due to the scalar product, it is necessary to adjust the physical constants:

1 We recall that $\nabla \varphi=\left(\begin{array}{l}\varphi, 1 \\ \varphi, 2 \\ \varphi, 3\end{array}\right)$. 


$$
\begin{array}{ll}
a_{I J}=\mathrm{a}_{i j k h} & \text { if } 1 \leq I, J \leq 3, \\
a_{I J}=\sqrt{2} \mathrm{a}_{i j k h} & \text { if } 1 \leq I, J \leq 3 \text { and } 4 \leq J, I \leq 6, \\
a_{I J}=2 \mathrm{a}_{i j k h} & \text { if } 4 \leq I, J \leq 6, \\
b_{I k}=\mathrm{b}_{i j k} & \text { if } 1 \leq I \leq 3 \\
b_{I k}=\sqrt{2} \mathrm{~b}_{i j k} & \text { if } 4 \leq I \leq 6, \\
c_{I k}=\mathrm{c}_{i j k} & \text { if } 1 \leq I \leq 3 \\
c_{I k}=\sqrt{2} \mathrm{c}_{i j k} & \text { if } 4 \leq I \leq 6
\end{array}
$$

The relation (3), which governs the linearly piezomagnetoelectric constitutive law, then takes the form

$$
\left(\begin{array}{c}
\sigma_{11} \\
\sigma_{22} \\
\sigma_{33} \\
\sqrt{2} \sigma_{23} \\
\sqrt{2} \sigma_{31} \\
\sqrt{2} \sigma_{12} \\
D_{1} \\
D_{2} \\
D_{3} \\
B_{1} \\
B_{2} \\
B_{3}
\end{array}\right)=\left(\begin{array}{cccccc|ccc|ccc}
a_{11} & a_{12} & a_{13} & a_{14} & a_{15} & a_{16} & -b_{11} & -b_{12} & -b_{13} & -c_{11} & -c_{12}-c_{13} \\
a_{12} & a_{22} & a_{23} & a_{24} & a_{25} & a_{26} & -b_{21} & -b_{22} & -b_{23} & -c_{21} & -c_{22}-c_{23} \\
a_{13} & a_{23} & a_{33} & a_{34} & a_{35} & a_{36} & -b_{31} & -b_{32} & -b_{33} & -c_{31} & -c_{32} & -c_{33} \\
a_{14} & a_{24} & a_{34} & a_{44} & a_{45} & a_{46} & -b_{41} & -b_{42} & -b_{43} & -c_{41} & -c_{42}-c_{43} \\
a_{15} & a_{25} & a_{35} & a_{45} & a_{55} & a_{56} & -b_{51} & -b_{52} & -b_{53} & -c_{51} & -c_{52}-c_{53} \\
a_{61} & a_{62} & a_{63} & a_{64} & a_{65} & a_{66} & -b_{61} & -b_{62} & -b_{63} & -c_{61} & -c_{62}-c_{63} \\
\hline b_{11} & b_{21} & b_{31} & b_{41} & b_{51} & b_{61} & d_{11} & d_{12} & d_{13} & e_{11} & e_{12} & e_{13} \\
b_{12} & b_{22} & b_{32} & b_{42} & b_{52} & b_{62} & d_{12} & d_{22} & d_{23} & e_{21} & e_{22} & e_{23} \\
b_{13} & b_{23} & b_{33} & b_{43} & b_{53} & b_{63} & d_{13} & d_{23} & d_{33} & e_{31} & e_{32} & e_{33} \\
\hline c_{11} & c_{21} & c_{31} & c_{41} & c_{51} & c_{61} & e_{11} & e_{21} & e_{31} & f_{11} & f_{12} & f_{13} \\
c_{12} & c_{22} & c_{32} & c_{42} & c_{52} & c_{62} & e_{12} & e_{22} & e_{32} & f_{12} & f_{22} & f_{23} \\
c_{13} & c_{23} & c_{33} & c_{43} & c_{53} & c_{63} & e_{13} & e_{23} & e_{33} & f_{31} & f_{32} & f_{33}
\end{array}\right) \cdot\left(\begin{array}{c}
e_{11}(u) \\
e_{22}(u) \\
e_{33}(u) \\
\sqrt{2} e_{23}(u) \\
\sqrt{2} e_{31}(u) \\
\sqrt{2} e_{12}(u) \\
\varphi, 1 \\
\varphi_{, 2} \\
\varphi_{, 3} \\
\phi_{, 1} \\
\phi_{, 2} \\
\phi_{, 3}
\end{array}\right) .
$$

We therefore note that the generalized three dimensional kinematics of a piezomagnetoelectric solid is described by twelve variables (six mechanical, three electric and three magnetic).

Finally, we have the following boundary conditions on $S=\partial \Omega$ :

$$
\begin{array}{lll}
u_{i}=U_{i} & , & \sigma_{i j} n_{j}=F_{i} \\
\varphi=V^{e} & , & D_{i} n_{i}=Q^{e} \\
\phi=V^{m} & , & B_{i} n_{i}=Q^{m}
\end{array}
$$

We do not precise the associated partitions of $S$. Here $U_{i}, F_{i}, V^{e}, V^{m}, Q^{e}, Q^{m}$ and $n_{i}$ denote the specified mechanical displacement and surface force components, the electric and magnetic scalar potentials, the fluxes through $S$ of the electric and magnetic inductions, and the outward unit normal vector components, respectively.

The local three-dimensional piezomagnetoelectric problem $\mathcal{P}_{3 D}(\Omega)$ consists in finding the piezomagnetoelectric state $s=(u, \varphi, \phi)$ satisfying equations (1), (2) and (6). 


\subsection{Variational equations}

It is convenient to rewrite $\mathcal{P}_{3 D}(\Omega)$ in another form in order to gather useful informations on the piezomagnetoelectric state $s=(u, \varphi, \phi)$. Multiplying by sufficiently $\operatorname{smooth}^{2}$ kinematically admissible virtual displacements $v_{i}$, electric potential $\psi$ and magnetic potential $\Psi$, equation (1) becomes equivalent to

$$
\int_{\Omega}\left(\sigma_{i j, j}+f_{i}\right) v_{i} d \Omega+\int_{\Omega} D_{i, i} \psi d \Omega+\int_{\Omega} B_{i, i} \Psi d \Omega=0 .
$$

Integrating by part this expression leads to

$$
\begin{aligned}
& -\int_{\Omega} \sigma_{i j, j} v_{i, j} d \Omega+\int_{S} \sigma_{i j} n_{j} v_{i} d S+\int_{\Omega} f_{i} v_{i} d \Omega \\
& \quad-\int_{\Omega} D_{i} \psi_{, i} d \Omega+\int_{S} D_{i} n_{i} \psi d \Omega-\int_{\Omega} B_{i} \Psi_{, i} d \Omega+\int_{S} B_{i} n_{i} \Psi d \Omega=0 .
\end{aligned}
$$

The symmetry of the stress tensor $\left(\sigma_{i j}=\sigma_{j i}\right)$, the boundary conditions (6) together with the definition of $e_{i j}(u)$ then give

$$
\begin{aligned}
& -\int_{\Omega} \sigma_{i j, j} e_{i j}(v) d \Omega+\int_{S} F_{i} v_{i} d S+\int_{\Omega} f_{i} v_{i} d \Omega \\
& \quad-\int_{\Omega} D_{i} \psi, i d \Omega+\int_{S} Q^{e} \psi d S-\int_{\Omega} B_{i} \Psi_{, i} d \Omega+\int_{S} Q^{m} \Psi d S=0 .
\end{aligned}
$$

We introduce the linear form $L$ such that

$$
L(r)=\int_{S} F_{i} v_{i} d S+\int_{\Omega} f_{i} v_{i} d \Omega+\int_{S} Q^{e} \psi d S+\int_{S} Q^{m} \Psi d S,
$$

for all kinematically admissible virtual piezomagnetoelectric state $r=(v, \psi, \Psi)$. We notice that

$$
-\int_{\Omega} \sigma_{i j, j} e_{i j}(v) d \Omega-\int_{\Omega} D_{i} \psi_{, i} d \Omega-\int_{\Omega} B_{i} \Psi_{, i} d \Omega=-\int_{\Omega} M\left(\begin{array}{c}
e(u) \\
\nabla \varphi \\
\nabla \phi
\end{array}\right) \cdot\left(\begin{array}{c}
e(v) \\
\nabla \psi \\
\nabla \Psi
\end{array}\right) d \Omega
$$

and, for brevity, define the bilinear form $m$ associated with the piezomagnetoelectric potential $\frac{1}{2}(\sigma \cdot e+D \cdot E+B \cdot H)$

$$
m(s, r)=m((u, \varphi, \phi),(v, \psi, \Psi))=\int_{\Omega} M\left(\begin{array}{c}
e(u) \\
\nabla \varphi \\
\nabla \phi
\end{array}\right) \cdot\left(\begin{array}{c}
e(v) \\
\nabla \psi \\
\nabla \Psi
\end{array}\right) d \Omega
$$

\footnotetext{
${ }^{2}$ In the sequel, we do not precise the exact mathematical background of such a formulation.
} 
It is then possible to reformulate the problem of determining the piezomagnetoelectric state at equilibrium through:

$\mathcal{P}_{3 D}(\Omega)\left\{\begin{array}{l}\text { Find } s=(u, \varphi, \phi) \text { sufficiently smooth such that } \\ m(s, r)=L(r), \text { for all virtual piezomagnetoelectric state } r=(v, \psi, \Psi) .\end{array}\right.$

This expression of the piezomagnetoelectric problem is at the starting point of either finite element formulations or mathematical questions in (linear) piezomagnetoelectricity.

\section{The problem of piezomagnetoelectric plates}

In the plate models derivation, a crucial role is played by the thickness direction (also called the outplane direction). For commodity, this direction corresponds to the third coordinate axis. The role played by the thickness is crucial because it is very small compared to the other dimensions of the plate : $\Omega=\omega \times(-\varepsilon / 2,+\varepsilon / 2)$, where $\omega$ is a bounded domain of $\mathbb{R}^{2}$ with smooth boundary and where $\varepsilon$ denotes the thickness of the plate. That leads to the idea of considering $\varepsilon$ as a small parameter and of connecting this parameter to the data of our problem, i.e. the piezomagnetoelectric coefficients, loading and state. In a sense, by this way, plate models can be interpreted as a peculiar piezomagnetoelectric state resulting of a given class of piezomagnetoelectric loading imposed to a thin flat piezomagnetoelectric plate.

From the mathematical point of view, the method consists in studying what does happen to the unique solution of $\mathcal{P}_{3 D}(\Omega)$ when $\Omega$ is the reference configuration of a flat piezoelectric body whose thickness goes to zero (this is the reason why this method belongs to the field of asymptotic analysis). The striking fact is that four models, i.e. four different kinds of behavior, appear at the limit. These four models are intimately connected to the type of electric and magnetic loadings. In order to emphasize on the fact that the models we get are arising through a dimension reduction process, they will be denoted by $\mathcal{P}_{2 D}(\Omega)$. More precisely, by different averagings through the thickness, it is possible to show that our limit models can be fully described by taking into account only the inplane coordinates.

In the sequel, we consider the following four piezomagnetoelectric boundary conditions on the set $\Gamma^{ \pm}$constituted by the lower and the upper faces of the flat thin plate occupying $\Omega$ :

$$
\begin{array}{ll}
(B C)_{1}: & D \cdot n=Q^{e^{ \pm}}, \quad B \cdot n=Q^{m^{ \pm}} \\
(B C)_{2}: & \varphi=V_{0}^{e^{ \pm}}, \quad \phi=V_{0}^{m^{ \pm}} \\
(B C)_{3}: & D \cdot n=Q^{e^{ \pm}}, \quad \phi=V_{0}^{m^{ \pm}}, \\
(B C)_{4}: & \varphi=V_{0}^{e^{ \pm}}, \quad B \cdot n=Q^{m^{ \pm}} .
\end{array}
$$

Here, we focus on the presentation of the obtained models. For the mathematical arguments underlying the whole analysis of this problem, and in particular for the details of the boundary conditions on the remaining part of $S$, we refer the reader to [13]. See also [10], [12] and [15]. 


\subsection{The sensor model}

Three kinds of information are needed to fully describe an piezomagnetoelectric model. These are the generalized kinematics (or generalized strain), the inner loading (or generalized stress) and the constitutive equations (which link them).

The generalized kinematics The generalized kinematics involves the tensor of small strains, the electric potential gradient and the magnetic potential gradient. In [13], we have shown that these three mathematical objects appear in reduced forms in the limit models. Here, we would like to emphasize on how they appear.

The displacements field We obtain a Kirchhoff-Love displacements field, which in particular means that the model cannot render shear effects. More precisely, a Kirchhoff-Love displacement $v$ satisfies:

$$
e(v)=\left(\begin{array}{ccc}
e_{11}(v) & e_{12}(v) & 0 \\
e_{12}(v) & e_{22}(v) & 0 \\
0 & 0 & 0
\end{array}\right)
$$

It is possible to show that Kirchhoff-Love displacements can be decomposed into a membrane and a flexural part (see [5], [12] and [15]). This result is a very classical one in pure elasticity. However, it is important to note that because of the symmetry of the small strain tensor, the number of purely mechanical variables comes down from six to three.

The electric and magnetic fields The asymptotic analysis of the three-dimensional problem shows a crucial difference between the actuator and the sensor cases: in the actuator case, the electric and magnetic fields intervene only (at the first order) through their outplane components while, in the sensor case, the electric and magnetic potentials do not depend (at the first order) on the outplane direction. Focusing here on the sensor case, we are in the situation for which the outplane direction plays no role, i.e. the electric and magnetic potentials do not depend on $x_{3}$ so that the limit model only takes into account four variables (the inplane electrical and magnetical ones).

We can therefore conclude that the limit generalized kinematics is described by seven variables instead of twelve in the full $3 D$ situation. It is represented by the $\widetilde{e}_{1}(u, \varphi, \phi)$ vector:

$$
\widetilde{e}_{1}(u, \varphi)=\left(\begin{array}{c}
e_{11}(u) \\
e_{22}(u) \\
\sqrt{2} e_{12}(u) \\
\varphi, 1 \\
\varphi_{, 2} \\
\phi_{, 1} \\
\phi_{, 2}
\end{array}\right)
$$


The generalized stress The generalized stress involves the stress tensor, the electric displacement and the magnetic induction vectors. As a result of the asymptotic analysis one finds that this mathematical object reduces to its inplane components, so that it takes the reduced form:

$$
\widetilde{\sigma}_{1}=\left(\begin{array}{c}
\sigma_{11} \\
\sigma_{22} \\
\sqrt{2} \sigma_{12} \\
D_{1} \\
D_{2} \\
B_{1} \\
B_{2}
\end{array}\right) .
$$

The sensor constitutive law We now have to identify the mathematical object $\widetilde{M}_{1}$ which link the generalized stress to the generalized strain that live on the plate. The algebraic arguments that lead to the exact formula of the limit constitutive law are presented and justified in [13], [12] and [15]. In fact, the limit constitutive equations emerge from a recombination of the piezomagnetoelectric components of $M$ in (5). This recombination is imposed by the structure of $\widetilde{e}_{1}(u, \varphi, \phi)$ and $\widetilde{\sigma}_{1}$ described above. In the sensor case, the recombination leads to rewrite (5) as

$$
\left(\begin{array}{c}
\sigma_{11} \\
\sigma_{22} \\
\sqrt{2} \sigma_{12} \\
D_{1} \\
D_{2} \\
B_{1} \\
B_{2} \\
\hline \sigma_{33} \\
\sqrt{2} \sigma_{23} \\
\sqrt{2} \sigma_{31} \\
D_{3} \\
B_{3}
\end{array}\right)=M_{1}^{\prime} \cdot\left(\begin{array}{c}
e_{11}(u) \\
e_{22}(u) \\
\sqrt{2} e_{12}(u) \\
\varphi_{, 1} \\
\varphi, 2 \\
\phi, 1 \\
\phi, 2 \\
\hline e_{33}(u) \\
\sqrt{2} e_{23}(u) \\
\sqrt{2} e_{31}(u) \\
\varphi, 3 \\
\phi, 3
\end{array}\right)
$$

with

$$
M_{1}^{\prime}=\left(\begin{array}{rrrrrrr|rrrrr}
a_{11} & a_{12} & a_{16} & -b_{11} & -b_{12} & -c_{11} & -c_{12} & a_{13} & a_{14} & a_{15} & -b_{13} & -c_{13} \\
a_{12} & a_{22} & a_{26} & -b_{21} & -b_{22} & -c_{21} & -c_{22} & a_{23} & a_{24} & a_{25} & -b_{23} & -c_{23} \\
a_{16} & a_{26} & a_{66} & -b_{61} & -b_{62} & -c_{61} & -c_{62} & a_{36} & a_{46} & a_{56} & -b_{63} & -c_{63} \\
b_{11} & b_{21} & b_{61} & d_{11} & d_{12} & e_{11} & e_{12} & b_{31} & b_{41} & b_{51} & d_{13} & e_{13} \\
b_{12} & b_{22} & b_{62} & d_{12} & d_{22} & e_{21} & e_{22} & b_{32} & b_{42} & b_{52} & d_{23} & e_{23} \\
c_{11} & c_{21} & c_{61} & e_{11} & e_{21} & f_{11} & f_{12} & c_{31} & c_{41} & c_{51} & e_{13} & f_{13} \\
c_{12} & c_{22} & c_{62} & e_{12} & e_{22} & f_{12} & f_{22} & c_{32} & c_{42} & c_{52} & e_{23} & f_{23} \\
\hline a_{13} & a_{23} & a_{36} & -b_{31} & -b_{32} & -c_{31} & -c_{32} & a_{33} & a_{34} & a_{35} & -b_{33} & -c_{33} \\
a_{14} & a_{24} & a_{46} & -b_{41} & -b_{42} & -c_{41} & -c_{42} & a_{34} & a_{44} & a_{45} & -b_{43} & -c_{43} \\
a_{15} & a_{25} & a_{56} & -b_{51} & -b_{52} & -c_{51} & -c_{52} & a_{35} & a_{45} & a_{55} & -b_{53} & -c_{53} \\
b_{13} & b_{23} & b_{63} & d_{13} & d_{23} & e_{13} & e_{23} & b_{33} & b_{43} & b_{53} & d_{33} & e_{33} \\
c_{13} & c_{23} & c_{63} & e_{13} & e_{23} & f_{13} & f_{23} & c_{33} & c_{43} & c_{53} & e_{33} & f_{33}
\end{array}\right) .
$$


Now, let

$$
\begin{aligned}
& M_{1}^{00}=\left(\begin{array}{rrrrrrr}
a_{11} & a_{12} & a_{16} & -b_{11} & -b_{12} & -c_{11} & -c_{12} \\
a_{12} & a_{22} & a_{26} & -b_{21} & -b_{22} & -c_{21} & -c_{22} \\
a_{16} & a_{26} & a_{66} & -b_{61} & -b_{62} & -c_{61} & -c_{62} \\
b_{11} & b_{21} & b_{61} & d_{11} & d_{12} & e_{11} & e_{12} \\
b_{12} & b_{22} & b_{62} & d_{12} & d_{22} & e_{21} & e_{22} \\
c_{11} & c_{21} & c_{61} & e_{11} & e_{21} & f_{11} & f_{12} \\
c_{12} & c_{22} & c_{62} & e_{12} & e_{22} & f_{12} & f_{22}
\end{array}\right), \\
& M_{1}^{0-}=\left(\begin{array}{rrrrr}
a_{13} & a_{14} & a_{15} & -b_{13} & -c_{13} \\
a_{23} & a_{24} & a_{25} & -b_{23} & -c_{23} \\
a_{36} & a_{46} & a_{56} & -b_{63} & -c_{63} \\
b_{31} & b_{41} & b_{51} & d_{13} & e_{13} \\
b_{32} & b_{42} & b_{52} & d_{23} & e_{23} \\
c_{31} & c_{41} & c_{51} & e_{13} & f_{13} \\
c_{32} & c_{42} & c_{52} & e_{23} & f_{23}
\end{array}\right) \\
& M_{1}^{-0}=\left(\begin{array}{rrrrrrr}
a_{13} & a_{23} & a_{36} & -b_{31} & -b_{32} & -c_{31} & -c_{32} \\
a_{14} & a_{24} & a_{46} & -b_{41} & -b_{42} & -c_{41} & -c_{42} \\
a_{15} & a_{25} & a_{56} & -b_{51} & -b_{52} & -c_{51} & -c_{52} \\
b_{13} & b_{23} & b_{63} & d_{13} & d_{23} & e_{13} & e_{23} \\
c_{13} & c_{23} & c_{63} & e_{13} & e_{23} & f_{13} & f_{23}
\end{array}\right), \\
& M_{1}^{--}=\left(\begin{array}{rrrrr}
a_{33} & a_{34} & a_{35} & -b_{33} & -c_{33} \\
a_{34} & a_{44} & a_{45} & -b_{43} & -c_{43} \\
a_{35} & a_{45} & a_{55} & -b_{53} & -c_{53} \\
b_{33} & b_{43} & b_{53} & d_{33} & e_{33} \\
c_{33} & c_{43} & c_{53} & e_{33} & f_{33}
\end{array}\right)
\end{aligned}
$$

Because the asymptotic analysis of $\mathcal{P}_{3 D}(\Omega)$ associated with the boundary conditions $(B C)_{1}$ shows that the vector $\left(\begin{array}{c}\sigma_{33} \\ \sqrt{2} \sigma_{23} \\ \sqrt{2} \sigma_{31} \\ D_{3} \\ B_{3}\end{array}\right)$ can be neglected, the sensor constitutive equations appears as the Schur complement (or the condensation) of the block $M_{1}^{--}$of $M_{1}^{\prime}$ :

$$
\widetilde{M}_{1}=M_{1}^{00}-M_{1}^{0-}\left(M_{1}^{--}\right)^{-1} M_{1}^{-0}
$$

Introducing the mechanical $(\mathrm{m})$, electrical (e) and magnetic (g) components of the generalized stress and strain, we associate to $\widetilde{M}_{1}$ the sub-operators $\widetilde{M}_{1_{\mathrm{mm}}}, \widetilde{M}_{1_{\mathrm{me}}}$, $\widetilde{M}_{1_{\mathrm{mg}}},, \widetilde{M}_{1_{\mathrm{em}}}, \widetilde{M}_{1_{\mathrm{ee}}}, \widetilde{M}_{1_{\mathrm{eg}}}, \widetilde{M}_{1_{\mathrm{gm}}}, \widetilde{M}_{1_{\mathrm{ge}}}$ and $\widetilde{M}_{1_{\mathrm{gg}}}$ :

$$
\widetilde{M}_{1}=\left(\begin{array}{ccc}
\widetilde{M}_{1 \mathrm{~mm}} & \widetilde{M}_{1_{\mathrm{me}}} & \widetilde{M}_{1 \mathrm{mg}} \\
\widetilde{M}_{1_{\mathrm{em}}} & \widetilde{M}_{1 \mathrm{ee}} & \widetilde{M}_{1_{\mathrm{eg}}} \\
\widetilde{M}_{1_{\mathrm{gm}}} & \widetilde{M}_{1_{\mathrm{ge}}} & \widetilde{M}_{1_{\mathrm{gg}}}
\end{array}\right)
$$


It is shown in [13] that $M$ and $\widetilde{M}_{1}$ share the same inner structure, that is:

$$
\begin{aligned}
& \widetilde{M}_{1_{\mathrm{mm}}}=\widetilde{M}_{1_{\mathrm{mm}}}^{T}, \widetilde{M}_{1_{\mathrm{me}}}=-\widetilde{M}_{1_{\mathrm{em}}}^{T}, \widetilde{M}_{1_{\mathrm{mg}}}=-\widetilde{M}_{1_{\mathrm{gm}}}^{T}, \\
& \widetilde{M}_{1_{\mathrm{ee}}}=\widetilde{M}_{1_{\mathrm{ee}}}^{T}, \widetilde{M}_{1_{\mathrm{eg}}}=\widetilde{M}_{1_{\mathrm{ge}}}^{T}, \widetilde{M}_{1_{\mathrm{gg}}}=\widetilde{M}_{1_{\mathrm{gg}}}^{T}
\end{aligned}
$$

Variational formulation of the sensor model Similarly to (12), we define

$$
\widetilde{m}_{1}(s, r)=\widetilde{m}_{1}((u, \varphi, \phi),(v, \psi, \Psi))=\int_{\Omega} \widetilde{M}_{1} \widetilde{e}_{1}(u, \varphi, \phi) \cdot \widetilde{e}_{1}(v, \psi, \Psi) d \Omega
$$

Our proposed model which allows us to determine the piezomagnetoelectric state of a plate-like sensor at equilibrium then reads as:

$\mathcal{P}_{2 D}^{1}(\Omega)\left\{\begin{array}{l}\text { Find } s=(u, \varphi, \phi) \text { sufficiently smooth such that } \\ \tilde{m}_{1}(s, r)=L(r), \text { for all virtual piezomagnetoelectric state } r=(v, \psi, \Psi) .\end{array}\right.$

As an asymptotic result, the thinner the plate (compared to its other dimensions), the more accurate the model. Mathematically speaking, it is of importance to precise that the function space on which live the (limit) admissible piezomagnetoelectric state is not the same as in the three-dimensional case. This is the reason why it is often spoken of "singular perturbations" problems.

Practically speaking, this case corresponds to a device which is able to measure (directly or indirectly) the fluxes of the electric and magnetic inductions, so that the linear form $L$ is perfectly determined. A numerical treatment of $\mathcal{P}_{2 D}^{1}(\Omega)$ then gives the piezomagnetoelectric state in the plate. That is why we can call this model sensor.

\subsection{The actuator model}

As it has been specified earlier, the difference between sensor and actuator models lies in the informations that the electric and magnetic potentials can take into account. Here, in the actuator case, these informations are collected only upon the outplane direction, while in the sensor case these informations were collected upon the two inplane directions. Of course, the purely mechanical informations do not change, but the fact that only $\varphi_{, 3}$ and $\phi_{, 3}$ appear in the actuator model radically changes the generalized kinematics and stress together with the constitutive law.

The generalized kinematics and stress As it has just been pointed out, displacements field is always of Kirchhoff-Love type (see (14)). As to the electric and magnetic potentials, it can be shown that only $E_{3}$ and $H_{3}$ appear. The generalized kinematics is therefore described by five variables at the limit. It is represented by the vector: 


$$
\widetilde{e}_{2}(u, \varphi)=\left(\begin{array}{c}
e_{11}(u) \\
e_{22}(u) \\
\sqrt{2} e_{12}(u) \\
\varphi, 3 \\
\phi, 3
\end{array}\right)
$$

Similarly, the generalized stress takes the form:

$$
\tilde{\sigma}_{2}=\left(\begin{array}{c}
\sigma_{11} \\
\sigma_{22} \\
\sqrt{2} \sigma_{12} \\
D_{3} \\
B_{3}
\end{array}\right)
$$

The actuator constitutive law The method that leads to the constitutive relations is similar to the one presented in the sensor case. However, the difficulty lies in an adequate piezomagnetoelectric coefficients recombination. We precise this point here.

First of all, we rewrite (5) as

$$
\left(\begin{array}{c}
\sigma_{11} \\
\sigma_{22} \\
\sqrt{2} \sigma_{12} \\
D_{3} \\
B_{3} \\
\hline \sigma_{33} \\
\sqrt{2} \sigma_{23} \\
\sqrt{2} \sigma_{31} \\
\hline D_{1} \\
D_{2} \\
B_{1} \\
B_{2}
\end{array}\right)=M_{2}^{\prime} \cdot\left(\begin{array}{c}
e_{11}(u) \\
e_{22}(u) \\
\sqrt{2} e_{12}(u) \\
\partial_{3} \varphi \\
\partial_{3} \phi \\
\hline e_{33}(u) \\
\sqrt{2} e_{23}(u) \\
\frac{\sqrt{2} e_{31}(u)}{\partial_{1} \varphi} \\
\partial_{2} \varphi \\
\partial_{1} \phi \\
\partial_{2} \phi
\end{array}\right)
$$

so that:

$$
M_{2}^{\prime}=\left(\begin{array}{rrrrr|rrr|rrrr}
a_{11} & a_{12} & a_{16} & -b_{13} & -c_{13} & a_{13} & a_{14} & a_{15} & -b_{11} & -b_{12} & -c_{11} & -c_{12} \\
a_{12} & a_{22} & a_{26} & -b_{23} & -c_{23} & a_{23} & a_{24} & a_{25} & -b_{21} & -b_{22} & -c_{21} & -c_{22} \\
a_{16} & a_{26} & a_{66} & -b_{63} & -c_{63} & a_{36} & a_{46} & a_{56} & -b_{61} & -b_{62} & -c_{61} & -c_{62} \\
b_{13} & b_{23} & b_{63} & d_{33} & e_{33} & b_{33} & b_{43} & b_{53} & d_{13} & d_{23} & e_{31} & e_{32} \\
c_{13} & c_{23} & c_{63} & e_{33} & f_{33} & c_{33} & c_{43} & c_{53} & e_{13} & e_{23} & f_{13} & f_{23} \\
\hline a_{13} & a_{23} & a_{36} & -b_{33} & -c_{33} & a_{33} & a_{34} & a_{35} & -b_{31} & -b_{32} & -c_{31} & -c_{32} \\
a_{14} & a_{24} & a_{46} & -b_{43} & -c_{43} & a_{34} & a_{44} & a_{45} & -b_{41} & -b_{42} & -c_{41} & -c_{42} \\
a_{15} & a_{25} & a_{56} & -b_{53} & -c_{53} & a_{35} & a_{45} & a_{55} & -b_{51} & -b_{52} & -c_{51} & -c_{52} \\
\hline b_{11} & b_{21} & b_{61} & d_{13} & e_{13} & b_{31} & b_{41} & b_{51} & d_{11} & d_{12} & e_{11} & e_{12} \\
b_{12} & b_{22} & b_{62} & d_{23} & e_{23} & b_{32} & b_{42} & b_{52} & d_{12} & d_{22} & e_{21} & e_{22} \\
c_{11} & c_{21} & c_{61} & e_{31} & f_{13} & c_{31} & c_{41} & c_{51} & e_{11} & e_{21} & f_{11} & f_{12} \\
c_{12} & c_{22} & c_{62} & e_{32} & f_{23} & c_{32} & c_{42} & c_{52} & e_{12} & e_{22} & f_{12} & f_{22}
\end{array}\right) .
$$


and define

$$
\begin{gathered}
M_{2}^{00}=\left(\begin{array}{lllrr}
a_{11} & a_{12} & a_{16} & -b_{13} & -c_{13} \\
a_{12} & a_{22} & a_{26} & -b_{23} & -c_{23} \\
a_{16} & a_{26} & a_{66} & -b_{63} & -c_{63} \\
b_{13} & b_{23} & b_{63} & d_{33} & e_{33} \\
c_{13} & c_{23} & c_{63} & e_{33} & f_{33}
\end{array}\right), M_{2}^{0-}=\left(\begin{array}{llll}
a_{13} & a_{14} & a_{15} \\
a_{23} & a_{24} & a_{25} \\
a_{36} & a_{46} & a_{56} \\
b_{33} & b_{43} & b_{53} \\
c_{33} & c_{43} & c_{53}
\end{array}\right), \\
M_{2}^{-0}=\left(\begin{array}{lllll}
a_{13} & a_{23} & a_{36} & -b_{33} & -c_{33} \\
a_{14} & a_{24} & a_{46} & -b_{43} & -c_{43} \\
a_{15} & a_{25} & a_{56} & -b_{53} & -c_{53}
\end{array}\right), M_{2}^{--}=\left(\begin{array}{lll}
a_{33} & a_{34} & a_{35} \\
a_{34} & a_{44} & a_{45} \\
a_{35} & a_{45} & a_{55}
\end{array}\right) .
\end{gathered}
$$

Here, the asymptotic analysis of $\mathcal{P}_{3 D}(\Omega)$ associated with the boundary conditions $(B C)_{2}$ shows that the three vectors $\left(\begin{array}{c}\sigma_{33} \\ \sqrt{2} \sigma_{23} \\ \sqrt{2} \sigma_{31}\end{array}\right),\left(\begin{array}{c}\partial_{1} \varphi \\ \partial_{2} \varphi\end{array}\right)$ and $\left(\begin{array}{c}\partial_{1} \phi \\ \partial_{2} \phi\end{array}\right)$ can be neglected, so that the actuator constitutive equations reads as

$$
\widetilde{M}_{2}=M_{2}^{00}-M_{2}^{0-}\left(M_{2}^{--}\right)^{-1} M_{2}^{-0}
$$

This operator shares the same structure and symmetry properties as those exhibited in (20)-(21).

Similarly to the sensor case, in order to get the variational formulation of the plate-like actuator problem, we define

$$
\widetilde{m}_{2}(s, r)=\widetilde{m}_{2}((u, \varphi, \phi),(v, \psi, \Psi))=\int_{\Omega} \widetilde{M}_{2} \widetilde{e}_{2}(u, \varphi, \phi) \cdot \widetilde{e}_{2}(v, \psi, \Psi) d \Omega,
$$

and the problem of determining the piezomagnetoelectric state of a plate-like actuator at equilibrium then takes the form:

$\mathcal{P}_{2 D}^{2}(\Omega)\left\{\begin{array}{l}\text { Find } s=(u, \varphi, \phi) \text { sufficiently smooth such that } \\ \widetilde{m}_{2}(s, r)=L(r), \text { for all virtual piezomagnetoelectric state } r=(v, \psi, \Psi),\end{array}\right.$

which is also a singularly perturbed problem.

Remark 2. To be more precise, in the expression of the model $\mathcal{P}_{2 D}^{2}(\Omega)$, the terms 'sufficiently smooth' mean that $s$ has to satisfy $(B C)_{2}$ while $r$ has to satisfy $(B C)_{2}$ with $V_{0}^{e^{ \pm}}=V_{0}^{m^{ \pm}}=0$, see [13] for the technical details.

This case corresponds to a device subjected to given magnetic and electric potentials at its boundary. A numerical treatment of $\mathcal{P}_{2 D}^{2}(\Omega)$ supplies the piezomagnetoelectric state in the plate. Therefore, the mechanical state can be controlled through electric and magnetic loading. That is why we call this model actuator. Let us recall that an electrode is sufficient to apply an electrical potential. On the contrary, the apparatus for assigning a magnetic potential is more complex (see [3]). 


\subsection{The senso-actuator model}

We have chosen this denomination for this model because the boundary conditions correspond to the plate which is a sensor from the electrical point of view and, in the same time, an actuator from the magnetic point of view (see relation $(B C)_{3}$ in (13)). This means that the electrical informations are collected upon the inplane directions, while the magnetic information is collected only upon the outplane direction.

The generalized kinematics and stress It can be shown that only $E_{1}, E_{2}$ and $H_{3}$ appear in the limit model. The generalized kinematics is therefore described by six variables at the limit. It is represented by the vector:

$$
\widetilde{e}_{3}(u, \varphi)=\left(\begin{array}{c}
e_{11}(u) \\
e_{22}(u) \\
\sqrt{2} e_{12}(u) \\
\varphi, 1 \\
\varphi_{, 2} \\
\phi, 3
\end{array}\right) .
$$

Similarly, the generalized stress takes the form:

$$
\widetilde{\sigma}_{3}=\left(\begin{array}{c}
\sigma_{11} \\
\sigma_{22} \\
\sqrt{2} \sigma_{12} \\
D_{1} \\
D_{2} \\
B_{3}
\end{array}\right)
$$

The senso-actuator constitutive law As previously, the relation (5) has to be rewrited:

$$
\left(\begin{array}{c}
\sigma_{11} \\
\sigma_{22} \\
\sqrt{2} \sigma_{12} \\
D_{1} \\
D_{2} \\
B_{3} \\
\hline \sigma_{33} \\
\sqrt{2} \sigma_{23} \\
\sqrt{2} \sigma_{31} \\
D_{3} \\
\hline B_{1} \\
B_{2}
\end{array}\right)=M_{3}^{\prime} \cdot\left(\begin{array}{c}
e_{11}(u) \\
e_{22}(u) \\
\sqrt{2} e_{12}(u) \\
\partial_{1} \varphi \\
\partial_{2} \varphi \\
\partial_{3} \phi \\
\hline e_{33}(u) \\
\sqrt{2} e_{23}(u) \\
\sqrt{2} e_{31}(u) \\
\partial_{3} \varphi \\
\hline \partial_{1} \phi \\
\partial_{2} \phi
\end{array}\right)
$$

so that: 


$$
M_{3}^{\prime}=\left(\begin{array}{rrrrrr|rrrr|rr}
a_{11} & a_{12} & a_{16} & -b_{11} & -b_{12} & -c_{13} & a_{13} & a_{14} & a_{15} & -b_{13} & -c_{11} & -c_{12} \\
a_{12} & a_{22} & a_{26} & -b_{21} & -b_{22} & -c_{23} & a_{23} & a_{24} & a_{25} & -b_{23} & -c_{21} & -c_{22} \\
a_{16} & a_{26} & a_{66} & -b_{61} & -b_{62} & -c_{63} & a_{36} & a_{46} & a_{56} & -b_{63} & -c_{61} & -c_{62} \\
b_{11} & b_{21} & b_{61} & d_{11} & d_{12} & e_{13} & b_{31} & b_{41} & b_{51} & d_{13} & e_{11} & e_{12} \\
b_{12} & b_{22} & b_{62} & d_{12} & d_{22} & e_{23} & b_{32} & b_{42} & b_{52} & d_{23} & e_{21} & e_{22} \\
c_{13} & c_{23} & c_{63} & e_{13} & e_{23} & f_{33} & c_{33} & c_{43} & c_{53} & e_{33} & f_{13} & f_{23} \\
\hline a_{13} & a_{23} & a_{36} & -b_{31} & -b_{32} & -c_{33} & a_{33} & a_{34} & a_{35} & -b_{33} & -c_{31} & -c_{32} \\
a_{14} & a_{24} & a_{46} & -b_{41} & -b_{42} & -c_{43} & a_{34} & a_{44} & a_{45} & -b_{43} & -c_{41} & -c_{42} \\
a_{15} & a_{25} & a_{56} & -b_{51} & -b_{52} & -c_{53} & a_{35} & a_{45} & a_{55} & -b_{53} & -c_{51} & -c_{52} \\
b_{13} & b_{23} & b_{63} & d_{13} & d_{23} & e_{33} & b_{33} & b_{43} & b_{53} & d_{33} & e_{31} & e_{32} \\
\hline c_{11} & c_{21} & c_{61} & e_{11} & e_{21} & f_{13} & c_{31} & c_{41} & c_{51} & e_{31} & f_{11} & f_{12} \\
c_{12} & c_{22} & c_{62} & e_{12} & e_{22} & f_{23} & c_{32} & c_{42} & c_{52} & e_{32} & f_{12} & f_{22}
\end{array}\right) .
$$

and define

$$
\begin{aligned}
& M_{3}^{00}=\left(\begin{array}{lllrrr}
a_{11} & a_{12} & a_{16} & -b_{11} & -b_{12} & -c_{13} \\
a_{12} & a_{22} & a_{26} & -b_{21} & -b_{22} & -c_{23} \\
a_{16} & a_{26} & a_{66} & -b_{61} & -b_{62} & -c_{63} \\
b_{11} & b_{21} & b_{61} & d_{11} & d_{12} & e_{13} \\
b_{12} & b_{22} & b_{62} & d_{12} & d_{22} & e_{23} \\
c_{13} & c_{23} & c_{63} & e_{13} & e_{23} & f_{33}
\end{array}\right), M_{3}^{0-}=\left(\begin{array}{rrrrrr}
a_{13} & a_{14} & a_{15} & -b_{13} \\
a_{23} & a_{24} & a_{25} & -b_{23} \\
a_{36} & a_{46} & a_{56} & -b_{63} \\
b_{31} & b_{41} & b_{51} & d_{13} \\
b_{32} & b_{42} & b_{52} & d_{23} \\
c_{33} & c_{43} & c_{53} & e_{33}
\end{array}\right), \\
& M_{3}^{-0}=\left(\begin{array}{llllll}
a_{13} & a_{23} & a_{36} & -b_{31} & -b_{32} & -c_{33} \\
a_{14} & a_{24} & a_{46} & -b_{41} & -b_{42} & -c_{43} \\
a_{15} & a_{25} & a_{56} & -b_{51} & -b_{52} & -c_{53} \\
b_{13} & b_{23} & b_{63} & d_{13} & d_{23} & e_{33}
\end{array}\right), M_{3}^{--}=M_{2}^{--} .
\end{aligned}
$$

Here, the asymptotic analysis of $\mathcal{P}_{3 D}(\Omega)$ associated with the boundary conditions $(B C)_{3}$ of $(13)$ shows that the vectors $\left(\begin{array}{c}\sigma_{33} \\ \sqrt{2} \sigma_{23} \\ \sqrt{2} \sigma_{31}\end{array}\right)$ and $\left(\begin{array}{c}E_{3} \\ H_{1} \\ H_{2}\end{array}\right)$ can be neglected, so that the actuator constitutive equation reads as

$$
\widetilde{M}_{3}=M_{3}^{00}-M_{3}^{0-}\left(M_{3}^{--}\right)^{-1} M_{3}^{-0}
$$

This operator shares the same structure and symmetry properties as those exhibited in (20)-(21).

Thus, introducing

$$
\widetilde{m}_{3}(s, r)=\widetilde{m}_{3}((u, \varphi, \phi),(v, \psi, \Psi))=\int_{\Omega} \widetilde{M}_{3} \widetilde{e}_{3}(u, \varphi, \phi) \cdot \widetilde{e}_{3}(v, \psi, \Psi) d \Omega,
$$

the problem of determining the piezomagnetoelectric state of a plate-like sensoactuator at equilibrium takes the form:

$\mathcal{P}_{2 D}^{3}(\Omega)\left\{\begin{array}{l}\text { Find } s=(u, \varphi, \phi) \text { sufficiently smooth such that } \\ \widetilde{m}_{3}(s, r)=L(r), \text { for all virtual piezomagnetoelectric state } r=(v, \psi, \Psi) .\end{array}\right.$ 
Remark 3. As previously, the terms 'sufficiently smooth' here mean that $\phi$ has to satisfy $\phi=V_{0}^{m^{ \pm}}$on $\Gamma^{ \pm}$while $\Psi$ vanishes on $\Gamma^{ \pm}$, see [13].

\subsection{The actuato-sensor model}

Here, the boundary conditions correspond to the plate which is an actuator from the electrical point of view and, in the same time, a sensor from the magnetic point of view (see relation $(B C)_{4}$ of $(13)$ ). This means that the electrical information is collected only upon the outplane direction, while the magnetic informations are collected upon the inplane direction.

The generalized kinematics and stress It is easily shown that only $E_{3}$, $H_{1}$ and $H_{2}$ appear in the limit model. The generalized kinematics is therefore also described by six variables at the limit. It is represented by the vector:

$$
\widetilde{e}_{4}(u, \varphi)=\left(\begin{array}{c}
e_{11}(u) \\
e_{22}(u) \\
\sqrt{2} e_{12}(u) \\
\varphi, 3 \\
\phi_{, 1} \\
\phi_{, 2}
\end{array}\right)
$$

Similarly, the generalized stress takes the form:

$$
\widetilde{\sigma}_{4}=\left(\begin{array}{c}
\sigma_{11} \\
\sigma_{22} \\
\sqrt{2} \sigma_{12} \\
D_{3} \\
B_{1} \\
B_{2}
\end{array}\right)
$$

The actuato-sensor constitutive law We rewrite (5) as

$$
\left(\begin{array}{c}
\sigma_{11} \\
\sigma_{22} \\
\sqrt{2} \sigma_{12} \\
D_{3} \\
B_{1} \\
B_{2} \\
\hline \sigma_{33} \\
\sqrt{2} \sigma_{23} \\
\sqrt{2} \sigma_{31} \\
B_{3} \\
\hline D_{1} \\
D_{2}
\end{array}\right)=M_{4}^{\prime} \cdot\left(\begin{array}{c}
e_{11}(u) \\
e_{22}(u) \\
\sqrt{2} e_{12}(u) \\
\partial_{3} \varphi \\
\partial_{1} \phi \\
\partial_{2} \phi \\
\hline e_{33}(u) \\
\sqrt{2} e_{23}(u) \\
\sqrt{2} e_{31}(u) \\
\partial_{3} \phi \\
\hline \partial_{1} \varphi \\
\partial_{2} \varphi
\end{array}\right),
$$


so that:

$$
M_{4}^{\prime}=\left(\begin{array}{rrrrrr|rrrr|rr}
a_{11} & a_{12} & a_{16} & -b_{13} & -c_{11} & -c_{12} & a_{13} & a_{14} & a_{15} & -c_{13} & -b_{11} & -b_{12} \\
a_{12} & a_{22} & a_{26} & -b_{23} & -c_{21} & -c_{22} & a_{23} & a_{24} & a_{25} & -c_{23} & -b_{21} & -b_{22} \\
a_{16} & a_{26} & a_{66} & -b_{63} & -c_{61} & -c_{62} & a_{36} & a_{46} & a_{56} & -c_{63} & -b_{61} & -b_{62} \\
b_{13} & b_{23} & b_{63} & d_{33} & e_{31} & e_{32} & b_{33} & b_{43} & b_{53} & e_{33} & d_{13} & d_{23} \\
c_{11} & c_{21} & c_{61} & e_{31} & f_{11} & f_{12} & c_{31} & c_{41} & c_{51} & f_{13} & e_{11} & e_{21} \\
c_{12} & c_{22} & c_{62} & e_{32} & f_{12} & f_{22} & c_{32} & c_{42} & c_{52} & f_{23} & e_{12} & e_{22} \\
\hline a_{13} & a_{23} & a_{36} & -b_{33} & -c_{31} & -c_{32} & a_{33} & a_{34} & a_{35} & -c_{33} & -b_{31} & -b_{32} \\
a_{14} & a_{24} & a_{46} & -b_{43} & -c_{41} & -c_{42} & a_{34} & a_{44} & a_{45} & -c_{43} & -b_{41} & -b_{42} \\
a_{15} & a_{25} & a_{56} & -b_{53} & -c_{51} & -c_{52} & a_{35} & a_{45} & a_{55} & -c_{53} & -b_{51} & -b_{52} \\
c_{13} & c_{23} & c_{63} & e_{33} & f_{13} & f_{23} & c_{33} & c_{43} & c_{53} & f_{13} & e_{13} & e_{23} \\
\hline b_{11} & b_{21} & b_{61} & d_{13} & e_{11} & e_{12} & b_{31} & b_{41} & b_{51} & e_{13} & d_{11} & d_{12} \\
b_{12} & b_{22} & b_{62} & d_{23} & e_{21} & e_{22} & b_{32} & b_{42} & b_{52} & e_{23} & d_{12} & d_{22}
\end{array}\right) .
$$

and define

$$
\begin{aligned}
& M_{4}^{00}=\left(\begin{array}{lllrrr}
a_{11} & a_{12} & a_{16} & -b_{13} & -c_{11} & -c_{12} \\
a_{12} & a_{22} & a_{26} & -b_{23} & -c_{21} & -c_{22} \\
a_{16} & a_{26} & a_{66} & -b_{63} & -c_{61} & -c_{62} \\
b_{13} & b_{23} & b_{63} & d_{33} & e_{31} & e_{32} \\
c_{11} & c_{21} & c_{61} & e_{31} & f_{11} & f_{12} \\
c_{12} & c_{22} & c_{62} & e_{32} & f_{12} & f_{22}
\end{array}\right), M_{4}^{0-}=\left(\begin{array}{lllll}
a_{13} & a_{14} & a_{15} & -c_{13} \\
a_{23} & a_{24} & a_{25} & -c_{23} \\
a_{36} & a_{46} & a_{56} & -c_{63} \\
b_{33} & b_{43} & b_{53} & e_{33} \\
c_{31} & c_{41} & c_{51} & f_{13} \\
c_{32} & c_{42} & c_{52} & f_{23}
\end{array}\right), \\
& M_{4}^{-0}=\left(\begin{array}{llllll}
a_{13} & a_{23} & a_{36} & -b_{33} & -c_{31} & -c_{32} \\
a_{14} & a_{24} & a_{46} & -b_{43} & -c_{41} & -c_{42} \\
a_{15} & a_{25} & a_{56} & -b_{53} & -c_{51} & -c_{52} \\
c_{13} & c_{23} & c_{63} & e_{33} & f_{13} & f_{23}
\end{array}\right), M_{4}^{--}=M_{3}^{--} .
\end{aligned}
$$

Because the vector $\left(\begin{array}{c}\sigma_{33} \\ \sqrt{2} \sigma_{23} \\ \sqrt{2} \sigma_{31} \\ E_{1} \\ E_{2} \\ H_{3}\end{array}\right)$ can be neglected, the actuato-sensor constitutive equation reads as

$$
\widetilde{M}_{4}=M_{4}^{00}-M_{4}^{0-}\left(M_{4}^{--}\right)^{-1} M_{4}^{-0}
$$

This operator shares the same structure and symmetry properties as those exhibited in (20)-(21).

We define

$$
\widetilde{m}_{4}(s, r)=\widetilde{m}_{4}((u, \varphi, \phi),(v, \psi, \Psi))=\int_{\Omega} \widetilde{M}_{4} \widetilde{e}_{4}(u, \varphi, \phi) \cdot \widetilde{e}_{4}(v, \psi, \Psi) d \Omega,
$$

and the problem of determining the piezomagnetoelectric state of a plate-like actuatosensor at equilibrium then takes the form: 
$\mathcal{P}_{2 D}^{4}(\Omega)\left\{\begin{array}{l}\text { Find } s=(u, \varphi, \phi) \text { sufficiently smooth such that } \\ \widetilde{m}_{4}(s, r)=L(r), \text { for all virtual piezomagnetoelectric state } r=(v, \psi, \Psi) .\end{array}\right.$

Remark 4. Here, the terms 'sufficiently smooth' mean that $\varphi$ has to satisfy $\varphi=V_{0}^{e^{ \pm}}$ on $\Gamma^{ \pm}$while $\psi$ vanishes on $\Gamma^{ \pm}$, see [13].

\section{Influence of crystalline symmetries}

It is interesting to give some properties of the operator $\widetilde{M}_{p}(p=1,2,3,4)$, which supplies the constitutive equations of the piezomagnetoelectric thin plates. As we saw, the fundamental coupling properties of $M$ remains true for $\widetilde{M}_{p}$ :

$$
\widetilde{M}_{p_{\mathrm{me}}}=-\left(\widetilde{M}_{p_{\mathrm{em}}}\right)^{T}, \quad \widetilde{M}_{p_{\mathrm{mg}}}=-\left(\widetilde{M}_{p_{\mathrm{gm}}}\right)^{T}, \quad \widetilde{M}_{p_{\mathrm{eg}}}=\left(\widetilde{M}_{p_{\mathrm{ge}}}\right)^{T}
$$

where $\mathrm{m}$, e and $\mathrm{g}$ respectively denote the mechanical, electric and magnetic components of the generalized kinematics and stress (see relation (20) for example).

It is important to note that in relation (2), $\mathrm{a}, \mathrm{b}, \mathrm{d}$, and $\mathrm{f}$ are polar tensors while $c$ and e are axial ones. Moreover, magnetic effects involve symmetry with respect to time. Recall that $\mathrm{a}, \mathrm{b}, \mathrm{d}$, and $\mathrm{f}$ are even tensors while $\mathrm{c}$ and $\mathrm{e}$ are odd ones with respect to time reversal. Of course, we restrict our study to materials that are at the same time piezoelectric and piezomagnetic. From the symmetry point of view, there are fourty five such crystal classes (see [9] for example). In the sequel, we will use the prime to denote time reversal. In the case of a polarization normal to the plate, we have the following properties ${ }^{3}$ :

- When $p=1, \widetilde{M}_{1}$ may be represented through Voigt notations by a $7 \times 7$ matrix. There is a structural switch off (that is: a piezoelectric and a piezomagnetic decoupling ; mathematically speaking: $\widetilde{M}_{1_{\mathrm{me}}}=\widetilde{M}_{1_{\mathrm{mg}}}=0$ ) for all the crystal classes of the orthorhombic, tetragonal and cubic systems. This also occurs in the monoclinic system for the class 2 and in the hexagonal system for the classes $6,622,62^{\prime} 2^{\prime}, 6 m m$ and $6 m^{\prime} m^{\prime}$. There are only ten crystal classes for which piezoelectric and piezomagnetic couplings simultaneously occur (i.e. $\widetilde{M}_{1_{\mathrm{me}}}$ and $\left.\widetilde{M}_{1_{\mathrm{mg}}} \neq 0\right): 1, m^{\prime}, 3, \overline{6}^{\prime}, 32,32^{\prime}, 3 m, 3 m^{\prime}, \overline{6}^{\prime} m 2^{\prime}$ and $\overline{6}^{\prime} m^{\prime} 2$.

- When $p=2, \widetilde{M}_{2}$ may be represented by a $5 \times 5$ matrix and $\widetilde{M}_{2_{\mathrm{mm}}}$ involves only mechanical terms. The crystal classes for which the piezoelectric and the piezomagnetic couplings simultaneously occur are: $1,2,3,4,4^{\prime}, \overline{4}, \overline{4}^{\prime}, 6,222$, $2^{\prime} 2^{\prime} 2,2 m m, 2 m^{\prime} m^{\prime}, 2^{\prime} m m^{\prime}, 3 m^{\prime}, 4 m^{\prime} m^{\prime}, 4^{\prime} m m^{\prime}, \overline{4} m^{\prime} 2^{\prime}, \overline{4}^{\prime} m 2^{\prime}, \overline{4}^{\prime} m^{\prime} 2,6 m^{\prime} m^{\prime}$, 23 and $\overline{4}^{\prime} 3 m^{\prime}$. Moreover, the structural swith-off occur with the classes $m^{\prime}, \overline{6}^{\prime}$, $32,422,622,6^{\prime} 22^{\prime}, \overline{6} m 2, \overline{6}^{\prime} m 2^{\prime}$ and $\overline{6}^{\prime} m^{\prime} 2$.

- When $p=3, \widetilde{M}_{3}$ may be represented by a $6 \times 6$ matrix. The structural swithoff occur with the classes $2^{\prime}, 6^{\prime}, 422,4 m m, \overline{4} m 2,622,6^{\prime} 22^{\prime}, 6 m m$ and $6^{\prime} m^{\prime} m$. There are only seven classes for which piezoelectric and piezomagnetic couplings

\footnotetext{
${ }^{3}$ From now on, the letter $m$ in Sans Serif font stands for 'mechanical' while the same letter $m$ in italic stands for 'mirror', as it is usually understood in crystallography.
} 
simultaneously occur: $1, m, 3, \overline{6}, 32^{\prime}, 3 m^{\prime}$ and $\overline{6} m^{\prime} 2^{\prime}$. Moreover, the electromagnetic coupling always vanishes (i.e. $\left.\widetilde{M}_{12_{e g}^{\varepsilon}}^{\varepsilon}=0\right)$, except for the classes $1,2^{\prime}$ and $m$.

- When $p=4, \widetilde{M}_{4}$ may be represented by a $6 \times 6$ matrix. The structural swithoff occur with the classes $m, \overline{6}, 422,42^{\prime} 2^{\prime}, 4^{\prime} 22^{\prime}, 622,62^{\prime} 2^{\prime}, \overline{6} m 2$ and $\overline{6} m^{\prime} 2^{\prime}$. There are only seven classes for which piezoelectric and piezomagnetic couplings simultaneously occur: $1,2^{\prime}, 3,6^{\prime}, 3 m, 3 m^{\prime}$ and $6^{\prime} m^{\prime} m$. Moreover, the electromagnetic coupling always vanishes, except for the classes $1,2^{\prime}$ and $m$.

- For the classes $m^{\prime}, \overline{6}^{\prime}, 32,422,622,6^{\prime} 22^{\prime}, \overline{6} m 2, \overline{6}^{\prime} m 2^{\prime}$ and $\overline{6}^{\prime} m^{\prime} 2$ (and only these), all the $\widetilde{M}_{p_{\mathrm{mm}}}$ are identical.

- For the classes $\overline{6}^{\prime}, \overline{6}^{\prime} m 2^{\prime}$ and $\overline{6}^{\prime} m^{\prime} 2$ when $p=1$ and for the classes $222, \overline{4}^{\prime}$, $\overline{4}^{\prime} 2^{\prime} m, \overline{4}^{\prime} 2 m^{\prime}, 23$ and $\overline{4}^{\prime} 3 m^{\prime}$ when $p=2$, the operators $\widetilde{M}_{p_{\mathrm{mm}}}, \widetilde{M}_{p_{\mathrm{me}}}, \widetilde{M}_{p_{\mathrm{mg}}}, \widetilde{M}_{p_{\mathrm{ee}}}$, $\widetilde{M}_{p_{\mathrm{eg}}}$ and $\widetilde{M}_{p_{\mathrm{gg}}}$ involve only mechanical, piezoelectric, piezomagnetic, dielectric, electromagnetic and magnetic permeability coefficients respectively, i.e. there is no mixing even if coupling always appears. In all other situations, these operators involve a mixture of coefficients of different types.

- $\widetilde{M}_{2_{\mathrm{mm}}}$ involves mechanical terms only,

- $\widetilde{M}_{1_{\mathrm{mm}}}=\widetilde{M}_{2_{\mathrm{mm}}}$ for the crystalline classes $m, 32,422, \overline{6}, 622$ and $\overline{6} m 2$,

- $\widetilde{M}_{1_{\mathrm{mm}}}$ involves electrical terms except for these previous classes,

- when $p=1$, there is an electromechanical decoupling $\left(\widetilde{M}_{p_{\text {me }}}=0\right)$ for the classes 2, 222, $2 m m, 4, \overline{4}, 422,4 m m, \overline{4} 2 m, 6,622,6 m m, 23$ and $\overline{4} 3 m$, when $p=2$, this decoupling occurs with the classes $m, 32,422, \overline{6}, 622$ and $\overline{6} m 2$, nevertheless the operators $\widetilde{M}_{p_{\mathrm{mm}}}$ and $\widetilde{M}_{p_{\mathrm{ee}}}$ involve a mixture of elastic, piezoelectric and dielectric coefficients. In these cases, the plate can be considered as no more piezoelectric. We are then in a situation of a structural switch off of the piezoelectric effect.

We then enlighten situations for which piezoelectric materials lead to nonpiezoelectric structures. For recent results concerning the reverse situation, that is the possibility of conceiving piezoelectric composites without using piezoelectric materials, the reader is refered to [8].

\section{Application and example: $4^{\prime} 22^{\prime}$ crystalline class}

We consider in this Section the case of a plate constituted by a material whose crystalline symmetry class is $4^{\prime} 22^{\prime}$. For this kind of material, it is possible to show (see [2] for example) that the constitutive relation (5) takes the form: 


$$
\left(\begin{array}{c}
\sigma_{11} \\
\sigma_{22} \\
\sigma_{33} \\
\sqrt{2} \sigma_{23} \\
\sqrt{2} \sigma_{31} \\
\sqrt{2} \sigma_{12} \\
D_{1} \\
D_{2} \\
D_{3} \\
B_{1} \\
B_{2} \\
B_{3}
\end{array}\right)=\left(\begin{array}{cccccc|ccc|ccc}
a_{11} & a_{12} & a_{13} & 0 & 0 & 0 & 0 & 0 & 0 & 0 & 0 & 0 \\
a_{12} & a_{11} & a_{13} & 0 & 0 & 0 & 0 & 0 & 0 & 0 & 0 & 0 \\
a_{13} & a_{13} & a_{33} & 0 & 0 & 0 & 0 & 0 & 0 & 0 & 0 & 0 \\
0 & 0 & 0 & a_{44} & 0 & 0 & -b_{41} & 0 & 0 & -c_{41} & 0 & 0 \\
0 & 0 & 0 & 0 & a_{44} & 0 & 0 & b_{41} & 0 & 0 & -c_{41} & 0 \\
0 & 0 & 0 & 0 & 0 & a_{66} & 0 & 0 & 0 & 0 & 0 & -c_{63} \\
\hline 0 & 0 & 0 & b_{41} & 0 & 0 & d_{11} & 0 & 0 & e_{11} & 0 & 0 \\
0 & 0 & 0 & 0 & -b_{41} & 0 & 0 & d_{11} & 0 & 0 & -e_{11} & 0 \\
0 & 0 & 0 & 0 & 0 & 0 & 0 & 0 & d_{33} & 0 & 0 & 0 \\
\hline 0 & 0 & 0 & c_{41} & 0 & 0 & e_{11} & 0 & 0 & f_{11} & 0 & 0 \\
0 & 0 & 0 & 0 & -c_{41} & 0 & 0 & e_{11} & 0 & 0 & f_{11} & 0 \\
0 & 0 & 0 & 0 & 0 & 0 & 0 & 0 & 0 & 0 & 0 & f_{33}
\end{array}\right) \cdot\left(\begin{array}{c}
e_{11}(u) \\
e_{22}(u) \\
e_{33}(u) \\
\sqrt{2} e_{23}(u) \\
\sqrt{2} e_{31}(u) \\
\sqrt{2} e_{12}(u) \\
\varphi, 1 \\
\varphi, 2 \\
\varphi, 3 \\
\phi_{, 1} \\
\phi, 2 \\
\phi, 3
\end{array}\right) .
$$

Because the simplest generalized kinematics corresponds to the actuator model, we begin with the illustration of the results presented in Section 3.2 in the case of a $4^{\prime} 22^{\prime}$ material. The boundary conditions are therefore those of $\left(B C_{2}\right)$ in $(13)$ and the relations (25)-(27) lead to:

$$
\left(\begin{array}{c}
\sigma_{11} \\
\sigma_{22} \\
\sqrt{2} \sigma_{12} \\
\hline D_{3} \\
\hline B_{3}
\end{array}\right)=\underbrace{\left(\begin{array}{ccc|c|c}
\left(a_{11} a_{33}-a_{13}^{2}\right) / a_{33} & \left(a_{12} a_{33}-a_{13}^{2}\right) / a_{33} & 0 & 0 & 0 \\
\left(a_{12} a_{33}-a_{13}^{2}\right) / a_{33} & \left(a_{11} a_{33}-a_{13}^{2}\right) / a_{33} & 0 & 0 & 0 \\
0 & 0 & a_{66} & 0 & -c_{63} \\
0 & 0 & 0 & d_{33} & 0 \\
\hline 0
\end{array}\right)}_{\widetilde{M}_{2}} \cdot\left(\begin{array}{c}
e_{11} \\
e_{22} \\
\frac{\sqrt{2} e_{12}}{\partial_{33}} \\
\hline \partial_{3} \\
\hline \partial_{3} \phi
\end{array}\right)
$$

We see that in its matrix form, the operator $\widetilde{M}_{2}$ can be decomposed in

$$
\begin{aligned}
\widetilde{M}_{2_{\mathrm{mm}}} & =\left(\begin{array}{ccc}
\left(a_{11} a_{33}-a_{13}^{2}\right) / a_{33} & \left(a_{12} a_{33}-a_{13}^{2}\right) / a_{33} & 0 \\
\left(a_{12} a_{33}-a_{13}^{2}\right) / a_{33} & \left(a_{11} a_{33}-a_{13}^{2}\right) / a_{33} & 0 \\
0 & 0 & a_{66}
\end{array}\right), \\
\widetilde{M}_{2_{\mathrm{me}}} & =\left(\begin{array}{l}
0 \\
0 \\
0
\end{array}\right), \quad \widetilde{M}_{2_{\mathrm{mg}}}=\left(\begin{array}{c}
0 \\
0 \\
-c_{63}
\end{array}\right), \\
\widetilde{M}_{2_{\mathrm{ee}}} & =d_{33}, \\
\widetilde{M}_{2_{\mathrm{eg}}} & =0 \\
\widetilde{M}_{2_{\mathrm{gg}}} & =f_{33} .
\end{aligned}
$$

We then observe that $\widetilde{M}_{2_{\mathrm{mm}}}$ is purely mechanical, $\widetilde{M}_{2_{\mathrm{mg}}}$ is purely piezomagnetic. Similarly, $\widetilde{M}_{2 \mathrm{ee}}$ and $\widetilde{M}_{2 \mathrm{gg}}$ are just composed of a dielectric and a magnetic permeability coefficient, respectively. Therefore, $\widetilde{M}_{2}$ is a not mixed operator. But, because 
$\widetilde{M}_{2_{\text {me }}}$ vanishes, the thin plate is no more piezoelectric, while still piezomagnetic and electromagnetic.

Now, let's consider the boundary condition $(B C)_{1}$ of $(13)$, which corresponds to the sensor situation. The generalized kinematics is in this case the richest one and the relations (17)-(19) lead to:

$$
\begin{aligned}
\widetilde{M}_{1_{\mathrm{mm}}} & =\left(\begin{array}{ccc}
\left(a_{11} a_{33}-a_{13}^{2}\right) / a_{33} & \left(a_{12} a_{33}-a_{13}^{2}\right) / a_{33} & 0 \\
\left(a_{12} a_{33}-a_{13}^{2}\right) / a_{33} & \left(a_{11} a_{33}-a_{13}^{2}\right) / a_{33} & 0 \\
0 & 0 & a_{66} f_{33}+c_{63}^{2} / f_{33}
\end{array}\right) \\
\widetilde{M}_{1_{\mathrm{me}}} & =\left(\begin{array}{cc}
0 & 0 \\
0 & 0 \\
0 & 0
\end{array}\right), \quad \widetilde{M}_{1_{\mathrm{mg}}}=\left(\begin{array}{ll}
0 & 0 \\
0 & 0 \\
0 & 0
\end{array}\right) \\
\widetilde{M}_{1_{\mathrm{ee}}} & =\left(\begin{array}{cc}
d_{11} a_{44}+b_{41}^{2} / a_{44} & 0 \\
0 & d_{11} a_{44}+b_{41}^{2} / a_{44}
\end{array}\right) \\
\widetilde{M}_{1_{\mathrm{eg}}} & =\left(\begin{array}{cc}
e_{11} a_{44}+b_{41} c_{41} / a_{44} & 0 \\
0 & e_{11} a_{44}+b_{41} c_{41} /-a_{44}
\end{array}\right) \\
\widetilde{M}_{1_{\mathrm{gg}}} & =\left(\begin{array}{cc}
f_{11} a_{44}+c_{41}^{2} / a_{44} & 0 \\
0 & f_{11} a_{44}+c_{41}^{2} / a_{44}
\end{array}\right)
\end{aligned}
$$

We firstly see that $\widetilde{M}_{1_{\mathrm{me}}}$ and $\widetilde{M}_{1_{\mathrm{mg}}}$ vanish, so that the plate is in this situation no more piezoelectric nor piezomagnetic: the structural switch off occurs. Moreover, all the other sub operators of $\widetilde{M}_{1}$ are mixed ones, even if $\widetilde{M}_{1_{\mathrm{mm}}}$ is very similar to $\widetilde{M}_{2_{\mathrm{mm}}}$, only the coefficients $\widetilde{M}_{1_{\mathrm{mm}} 3}$ and $\widetilde{M}_{2_{\mathrm{mm}} 3}$ being different.

Now, let's have a look at the results when we consider the boundary condition $(B C)_{3}$ of $(13)$, which corresponds to the senso-actuator situation. The relations (31)-(33) lead to:

$$
\begin{aligned}
& \widetilde{M}_{3_{\mathrm{mm}}}=\widetilde{M}_{2_{\mathrm{mm}}}, \widetilde{M}_{3_{\mathrm{me}}}=\left(\begin{array}{ll}
0 & 0 \\
0 & 0 \\
0 & 0
\end{array}\right), \widetilde{M}_{3_{\mathrm{mg}}}=\widetilde{M}_{1_{\mathrm{mg}}} \\
& \widetilde{M}_{3_{\mathrm{ee}}}=\widetilde{M}_{1_{\mathrm{ee}}}, \widetilde{M}_{3_{\mathrm{eg}}}=\left(\begin{array}{l}
0 \\
0
\end{array}\right), \widetilde{M}_{3_{\mathrm{gg}}}=\widetilde{M}_{2_{\mathrm{gg}}}
\end{aligned}
$$

Therefore, the operator $\widetilde{M}_{3}$ is mixed but the piezoelectric coupling disapears even if the thin plate is still piezomagnetic.

Finally, when the boundary conditions are those of a actuato-sensor, as specified in $(B C)_{4}$ of (13), the relations (37)-(39) lead to:

$$
\begin{aligned}
& \widetilde{M}_{4_{\mathrm{mm}}}=\widetilde{M}_{1_{\mathrm{mm}}}, \widetilde{M}_{4_{\mathrm{me}}}=\left(\begin{array}{l}
0 \\
0 \\
0
\end{array}\right), \widetilde{M}_{4_{\mathrm{me}}}=\left(\begin{array}{ll}
0 & 0 \\
0 & 0 \\
0 & 0
\end{array}\right), \\
& \widetilde{M}_{4_{\mathrm{ee}}}=\widetilde{M}_{2_{\mathrm{ee}}}, \widetilde{M}_{4_{\mathrm{eg}}}=\left(\begin{array}{ll}
0 & 0
\end{array}\right), \widetilde{M}_{4_{\mathrm{gg}}}=\widetilde{M}_{1_{\mathrm{gg}}} .
\end{aligned}
$$


In this operator, only $\widetilde{M}_{4 \mathrm{~mm}_{33}} \widetilde{M}_{4_{\mathrm{mm}} 5}$ and $\widetilde{M}_{4_{\mathrm{mm}} 66}$ are mixed. Anyway, all the coupling components vanish, so that the plate is no more piezoelectric, nor piezomagnetic, nor electromagnetic. It is another example of structural switch off.

\section{References}

1. Altay G. A., Cengiz Dökmeci M. (2005) Variational principles and vibrations of a functionally graded plate, Computers and Structures 83, 1340-1354

2. Bhagavantam S. (1966) Crystal symmetry and physical properties, Academic Press.

3. Bossavit A. (1988) Results for benchmark problem 5, the bath-cube experiment: an aluminium block in an alternating field, The International Journal for Computation and Mathematics in Electrical and Electronic Engineering, Vol. 7, Nos $1 \& 2,81-88$.

4. Chopra I. (2002) Review of State of Art of Smart Structures and Integrated Systems, AIAA Journal, 40, 11, 2145-2187.

5. Ciarlet P. G. (1997) Mathematical Elasticity, Vol. II. North Holland.

6. Eringen A. C. (1989) Theory of electromagnetic elastic plates, Int. J. Eng. Sci., $27(4), 363-375$.

7. M. Fiebig (2005), Revival of the magnetoelectric effect, J. Phys. D: Appl. Phys., Vol. 38, R123-R152.

8. Sharma N. D., Maranganti R., Sharma P. (2007) On the possibility of piezoelectric nanocomposites without using piezoelectric materials. J. Mech. Phys. Solids, $55,2328-2350$.

9. J. Sivardière, La symétrie en mathématiques, physique et chimie, Presses Universitaires de Grenoble, 1995.

10. Weller T., Licht C. (2001) Analyse asymptotique de plaques minces linéairement piézoélectriques. C. R. Acad. Sci. Paris, 333, Série I, 267-270.

11. Weller T., Licht C. (2004) Réponse dynamique asymptotique de plaques minces linéairement piézoélectriques dans l'approximation quasi-électrostatique, C. R. Mécanique, 332, 519-524.

12. Weller T. (2004) Etudes des symétries et modèles de plaques en piézoélectricité linéarisée. Thèse, Université Montpellier II, France.

13. Weller T., Licht C. (2007) Modeling of linearly electromagneto-elastic thin plates, C. R. Mécanique, 335, 201-206.

14. Weller T., Licht C. (2008) Asymptotic modeling of linearly piezoelectric slender rods, C. R. Mécanique, 336, 572-577.

15. Weller T., Licht C. Asymptotic modeling of thin piezoelectric plates, submitted.

16. Geymonat G., Licht C., Weller T. Plates made of piezoelectric materials: when are they really piezoelectric?, to appear in Applied Mathematical Modelling.

17. Yang J. (2006) The Mechanics of Piezoelectric Structures, World Scientific.

18. Zhang C. L., Chen W. Q., Li J. Y. and Yang J. S. (2009) Two-dimensional analysis of magnetoelectric effects in multiferroic laminated plates, IEEE Trans. Ultrason., Ferroelect., Freq. Contr., 56(5), 1046-1053. 